\title{
An unusual case of intestinal obstruction
}

\section{Giuseppe S. Sica*, Andrea Divizia, Emanuele Picone, Achille L. Gaspari}

Department of Surgery, Policlinico Tor Vergata, Tor Vergata University, Rome, Italy

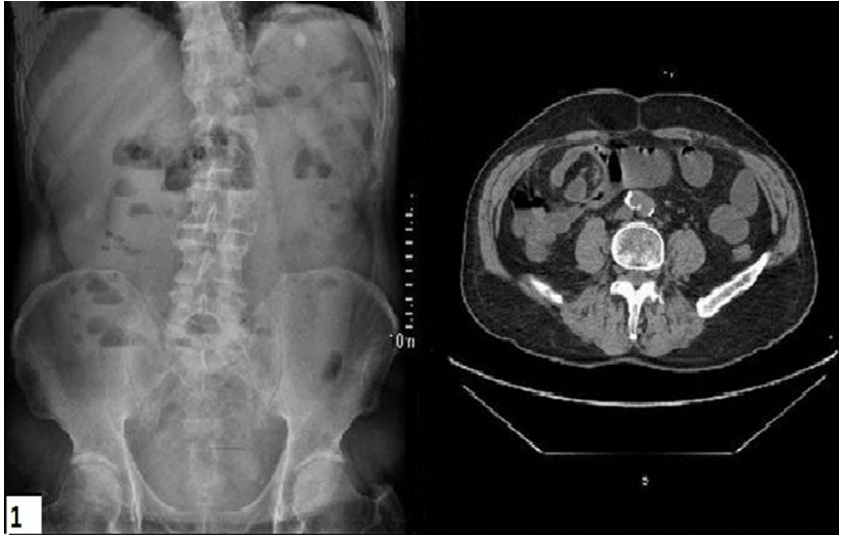

Fig. 1.

A 66 year-old man presented to emergency room complaining of abdominal pain, vomiting, and inactive bowel, and was admitted to hospital with a diagnosis of acute bowel obstruction. He had no history of previous abdominal surgery however, three years prior he had undergone excision of a thoracic skin melanoma, with right axillary lymph node dissection. The procedure was followed by 12 months of interferon therapy. Recent scheduled laboratory follow-up revealed an increase in inflammatory markers and mild anaemia. Abdominal X-ray showed diffusion of intestinal air-fluid levels and computed tomography scans suggested volvulus of the small bowel (Fig. 1).

At exploratory laparoscopy a duplication of the terminal ileum was found, and resection of $30 \mathrm{~cm}$ of ileum and primary anastomosis was performed. Pathology confirmed the cystic

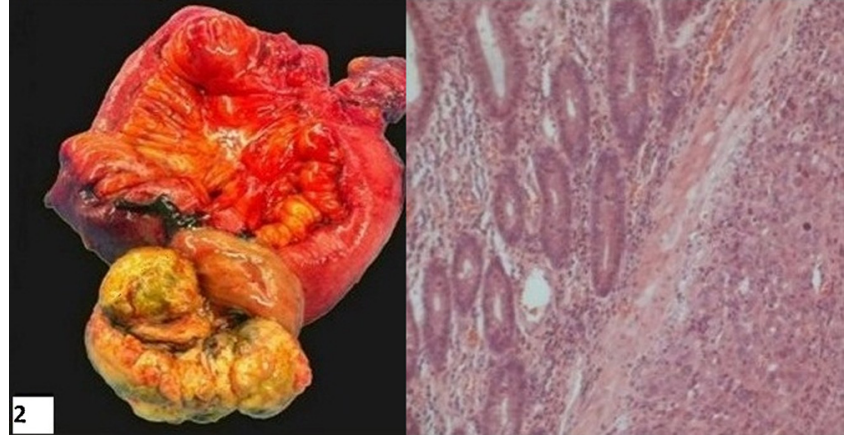

Fig. 2.

duplication of the terminal ileum, and a large polyp measuring $8.5 \mathrm{~cm} \times 5 \mathrm{~cm} \times 6 \mathrm{~cm}$ with a central green necrotic area (Fig. 2) was found on the mucosal surface. Immunohistochemical analysis revealed immunoreactivity for Melan A, S 100 and Vimentin, thereby confirming the presence of metastatic melanoma.

Roughly $80 \%$ of duplications of the intestine are found in infants, with the ileum as the most frequently affected site [1]. In adulthood, surgical resection should be considered in symptomatic patients. Intestinal metastases from primary melanoma are infrequent but can cause bowel obstruction, and relationship with the intestinal duplication is unknown.

\section{Reference}

[1] Ladd WE. Surgical diseases of the alimentary tract in infants. The New England Journal of Medicine 1936;215:705-8.

\footnotetext{
* Corresponding author at: Department of Surgery, Torre 8 I Piano, Policlinico Tor Vergata, Viale Oxford 81, 00133 Rome, Italy. Tel.: +39 0620903596; fax: +390620902926.

E-mail address: sigisica@gmail.com (G.S. Sica).
} 